\title{
ESTUDO NORMATIVO DA BATERIA PIAGET-HEAD PARA CRIANÇAS DA CIDADE DE CURITIBA: DADOS PRELIMINARES
}

\section{Standardization of the Piaget-Head Battery for children of Curitiba city: preliminary data.}

\author{
Plínio Marco de Toni ${ }^{1}$ \\ Egídio José Romanelli ${ }^{2}$ \\ Caroline Guisantes de Salvo ${ }^{3}$
}

\section{Resumo}

A presente pesquisa teve como objetivo realizar um estudo normativo preliminar dos subtestes Piaget 1, 2 e 3 (Bateria Piaget-Head) para crianças e adolescentes da cidade de Curitiba-PR. A Bateria Piaget-Head é utilizada na avaliação neuropsicológica e pretende avaliaro desenvolvimento da orientação direita-esquerda. A escolha deste instrumento é relevante por três fatores: a orientação direita-esquerda está relacionada a problemas de aprendizagem; a Bateria Piaget-Head é um dos poucos instrumentos utilizados no Brasil para a avaliação da orientação direita-esquerda; desde a construção do instrumento em 1950 na França, nenhuma normatização foi realizada para a realidade brasileira. Fizeram parte da amostra 102 crianças de 7 a 13 anos, de ambos os sexos, matriculados em escolas públicas e particulares de Curitiba. Os resultados não apontam diferenças nos escores entre os gêneros, escolas públicas e particulares e destros e canhotos. Verificou-se correlação positiva significativa entre itens pares e ímpares do subteste Piaget 3, evidenciando boa consistência interna do subteste. Foram realizadas tabelas de percentis por idade, além de uma comparação dos resultados encontrados com a normatização francesa.

Palavras-chave: Testes psicológicos, orientação direita-esquerda, Bateria Piaget-Head.

\section{Abstract}

The aim of this research was the standardization of the Piaget 1, 2, 3 subtests (Piaget-Head Battery) for the city of Curitiba-PR. The Piaget-Head Battery is used in neuropsychological assessment and aims to evaluate the development of the left-right orientation. This option for this instrument is relevant based in thee factors: the left-right orientation is related with leaning problems; the Piaget-Head Test is one of the few instruments used in Brazil for the left-right evaluation; since of development of this instrument in 1950 in France, any kind of standardization was created for the Brazilian reality. This sample used 102 children with age ranging from 7 to 13 years, both sex, matriculated in public and private schools in Curitiba. The results no not show differences in score between gender, public and private schools and right- and left-handed subjects. A positive correlation was found between even and odd itens in the Piaget 3 subtest, demonstrating a good internal consistence in the subtest. Percentile's tables were created by age criteria, and the results were compared with French standardization.

Keywords: Psychological tests, left-right orientation, Piaget-Head Battery.

1 Doutorando em Psicologia Escolar e Desenvolvimento Humano pela USP. Mestre em Psicologia da Infância e Adolescência pela UFPR. Professor adjunto da Faculdade Evangélica do Paraná, Universidade Tuiuti do Paraná e Faculdade Dom Bosco.

2 Pós-doutor em Neuropsicologia pela Universitè de Montreal, Canadá. Professor da Pós-graduação em Educação da Universidade Federal do Paraná.

3 Mestranda em Psicologia Clínica pela USP. Psicóloga e Bacharel em Psicologia pela UFPR.

Endereço para contato: Rua Marechal Deodoro, n. 1014, ap. 121 - Centro, Curitiba - PR. CEP 80060-010

E-mail: pliniomarco@yahoo.com.br 


\section{Introdução}

A história da neuropsicologia sugere um desenvolvimento tardio das baterias de testes direcionadas ao exame infantil em comparação àquelas próprias à população adulta. Ao contrário de baterias infantis, registros de testes neuropsicológicos para adultos são encontrados anteriormente a meados do século XX. Em 1941, Goldstein criou uma bateria específica para o funcionamento cognitivo adulto (Lefèvre, 1998). Em compensação, o desenvolvimento de baterias infantis tem seu início recente. De acordo com Simões (2002), “a elaboração significativa de novos testes, específicos para o exame neuropsicológico de crianças e adolescentes, ocorre apenas no final da década de 80 e, sobretudo, na década de $90 "$ ".

Isto representa um grande déficit nesta área do conhecimento, já que uma das principais populações beneficiadas pela avaliação neuropsicológica e, consequentemente, pelo uso da psicometria, é a infantil. Como salientado por Riechi (1996), todo o âmbito escolar, desde os primeiros processos de inserção escolar da criança, passando pela alfabetização e sua continuidade, é marcado fortemente pelo problema de aprendizagem. E, nesse sentido, o uso da neuropsicologia tem se revelado produtivo, com a emergência de uma nova área de atuação conhecida como neuropsicopedagogia (Riechi, Kruszielski, Marins \& De Toni, 2002). No entanto, para o diagnóstico preciso de problemas de aprendizagem, deve-se necessariamente conhecer a norma, o processo natural de desenvolvimento. Como salienta Hutt \& Hutt (1974), não é interessante estudar variações de comportamento a não ser que se conheça antes a norma da qual discrepam as variantes.

A dificuldade em desenvolver testes psicológicos para a população infantil e a escassez de material disponível para este fim têm como conseqüência a utilização de instrumentos não padronizados, simplesmente traduzidos e com tabelas normativas estrangeiras, fora da realidade brasileira. Noronha \& Alchieri (2002) salientam que, por muitos anos, psicólogos brasileiros usaram como recursos de avaliação instrumentos estrangeiros que não possuíam nenhum estudo normativo com amostras brasileiras.

De acordo com Kroeff (1988, p. 12-13),
Essa prática, de utilizar normas estrangeiras para a população nacional, é comum em nosso meio, e só é tolerada pela quase total inexistência de normas locais. O preço que pagamos por tais práticas é a insegurança em nossas conclusões... a imaturidade para um programa pode ser a maturidade suficiente para outro.

O interesse em desenvolver normatizações regionais para testes estrangeiros utilizados no Brasil é recente. Com o objetivo de manter a confiabilidade dos instrumentos, a legislação atual do Conselho Federal de Psicologia restringe o uso de testes psicológicos, definido normas de padronização e fixando prazos de no máximo 10 anos para a revisão deles (Conselho Federal de Psicologia, 2003). Mesmo assim, como apontado por Simões (2002), com freqüência os neuropsicólogos confiam na natureza "clínica" dos testes e não prestam devida atenção às propriedades psicométricas deles.

No campo da avaliação neuropsicológica, um dos instrumentos que vem sendo utilizado com freqüência é a Bateria Piaget-Head. Como apontado por Zazzo (1981), a Bateria Piaget-Head tem como proposta verificar o grau de desenvolvimento da orientação direita-esquerda em crianças e adolescentes. A bateria é composta por duas partes distintas, que correspondem cada qual a compilação de testes anteriores: Piaget "direita-esquerda" e Head "mão-olho-orelha". A primeira parte diz respeito a consignas que objetivam verificar a orientação direita-esquerda em suas diferentes formas: em si próprio (Piaget 1); no outro (Piaget 2); entre os objetos (Piaget 3). Na segunda parte é solicitada ao sujeito a reprodução de movimentos lateralizados, envolvendo desde a execução por ordem verbal (por exemplo: coloque sua mão esquerda na sua orelha direita) até a execução por reprodução direta (o examinador como modelo) e o uso de figuras esquemáticas. A pontuação se dá considerando as categorias: acerto, erro seguido de acerto, imagem especular, mão certa e orelha/ olho errados (ou vice-versa). As normas são fornecidas por idade.

A escolha deste instrumento de avaliação para a presente pesquisa é relevante por três motivos principais: a orientação direita-esquerda está diretamente relacionada com problemas de aprendizagem, dentre os quais a dislexia, a disgrafia e a discalculia (Fonseca, 1995; Pamplona Mo- 
rais, 1992; Luria, 1981); a Bateria Piaget-Head é um dos únicos instrumentos utilizados no Brasil para a avaliação neuropsicológica da orientação direita-esquerda ${ }^{1}$; a Bateria Piaget-Head foi desenvolvida por Galifret-Granjon em 1950 e padronizada com amostragem francesa (Zazzo, 1981). Desde então, não foi realizado nenhum estudo metodológico com o objetivo de normatizar o instrumento para a realidade brasileira ${ }^{2}$.

Considerando isto, a presente pesquisa tem como objetivo realizar um estudo normativo preliminar dos subtestes Piaget 1, 2 e 3 da Bateria Piaget-Head para crianças e adolescentes da cidade de Curitiba, traçando a primeira curva de desenvolvimento da orientação direita-esquerda para a população brasileira.

\section{Método}

\section{Participantes}

Foi estabelecida uma amostra de 102 alunos entre 7 anos e 0 meses e 13 anos e 11 meses, de ambos os sexos (53\% masculino, $47 \%$ feminino), matriculados na rede pública ou particular de ensino do município de Curitiba. A técnica de amostragem aleatória sistemática utilizada para a consolidação da amostra resultou em uma seleção não tendenciosa no que diz respeito ao gênero dos participantes $\left(\mathrm{X}^{2}=0,35 ; \mathrm{p}=0,55\right)$ e ao tipo de escola (pública ou particular) na qual estão matriculados $\left(X^{2}=0,35 ; \mathrm{p}=0,55\right)$. A proporção de canhotos encontrada na amostra de normatização $(4,5 \%)$ é comparável a estudos anteriores (8\% de canhotos em Pamplona Morais, 1992).

\section{Instrumentos}

Ficha de coleta de dados para descrição da amostra, considerando as variáveis gênero, idade, tipo de escola (pública e particular), preferên- cia manual e escolaridade, além da versão da Bateria Piaget-Head referenciada em Zazzo (1981).

\section{Procedimentos}

Os colégios que fizeram parte da amostra foram eleitos por conveniência, de acordo com a facilidade de cada auxiliar de pesquisa. Uma vez escolhido o colégio, para compor a amostra, fezse uso das listas de chamada das turmas compostas por alunos da faixa etária em questão, selecionando-os por meio de uma técnica de amostragem aleatória sistemática. Recrutou-se, assim, o primeiro aluno da lista alfabética de chamada e os demais por um intervalo de seleção preestabelecido. Os subtestes Piaget 1, Piaget 2 e Piaget 3 da Bateria Piaget-Head foram aplicados nos alunos, individualmente, em ambiente escolar.

\section{Tra ta mento dos dados}

Após pesquisa de campo, os dados extraídos da amostra de normatização passaram por três procedimentos:

1. Análise estatística, com utilização do software SPSS 11.0 for Windows (SPSS, Inc., 1994): caracterização da amostra de normatização; estatística descritiva do desempenho nos subtestes para as diferentes faixas etárias; comparação entre escolas, gêneros e lateralidade na amostra; análise de precisão pelo método das duas metades (correlação Produto-Momento de Pearson).

2. Confecção da tabela normativa percentilar correspondente às faixas etárias.

3. Comparação dos dados normativos obtidos pela presente pesquisa com a normatização francesa, pelo gráfico em linha. A normatização francesa da década de 50 omite, na publicação dos resultados (Zazzo, 1981), quaisquer referências aos dados brutos. Por isso, torna-se inviável uma análise estatística comparando os dois estudos de normatização.

1 Outros testes utilizados no Brasil para a avaliação da orientação direita-esquerda são: Bateria Psicomotora (Fonseca, 1995b); Bateria Neuropsicológica Luria-Christensen (Christensen, 1978); Teste de Imitação de Gestos (Bergès \& Lézine, 1987). Nenhum destes possui tabelas normativas para a população brasileira.

2 Não foram encontradas quaisquer referências à Bateria Piaget-Head em pesquisas realizadas no indexador OVID com as palavras-chaves "Piaget-Head Battery" e "left-right orientation". Buscas no PsycINFO indicaram que desde a criação da bateria até janeiro de 2004 foram publicados somente dois artigos sobre o teste no periódico espanhol Infancia y Aprendizaje (Forner, 1983a; Forner 1983b). 
Apresentação e a ná lise dos resultados

Considerando o formato como os subtestes Piaget 1, 2 e 3 da Bateria Piaget-Head foram construídos, o risco de acerto por tentativa e erro é considerável (50\% em cada consigna), como adverte Zazzo (1981). Isto justifica um escore global para os subtestes, como proposto neste estudo. Quando se totaliza a pontuação de 10 itens (Piaget 1, 2 e 3), uma variação de alguns pontos (devidos ao acaso) não incide diretamente sobre o escore global. Desta forma, o escore global torna-se um bom indicativo para a construção da curva de desenvolvimento da orientação direita-esquerda.
Analisando o escore Piaget 1-2-3, não foram observadas diferenças estatisticamente significativas $(\alpha=0,01)$ entre os desempenhos de crianças de escolas públicas e particulares $(t=-0,05 ; g l=100 ; p=0,96)$, entre os gêneros feminino e masculino $(\mathrm{t}=-0,20 ; \mathrm{gl}=100 ; \mathrm{p}=$ $0,85)$ e entre destros e canhotos $(\mathrm{t}=1,22 ; \mathrm{gl}=$ $5,69 ; \mathrm{p}=0,27)$. Estas análises permitem concluir a viabilidade de se agrupar todos os dados em uma mesma categoria. A Tabela 1 exprime as estatísticas descritivas para o escore Piaget 1-2-3, em função da idade, desconsiderando diferenças de gêneros, tipos de escola e lateralidade.

\section{Tabela 1. Estatística descritiva para o Escore Piaget 1-2-3.}

\begin{tabular}{|c|c|c|c|c|c|c|c|c|c|c|}
\hline \multirow{2}{*}{ Idade } & \multirow{2}{*}{$\mathrm{N}$} & \multirow{2}{*}{ Média } & \multirow{2}{*}{$\begin{array}{l}\text { Desvio } \\
\text { Padrão }\end{array}$} & \multirow{2}{*}{$\begin{array}{c}\text { Erro } \\
\text { Padrão } \\
\text { da Média }\end{array}$} & \multicolumn{2}{|c|}{$\begin{array}{c}\text { Intervalo de Confiança } \\
\alpha=0,05\end{array}$} & \multirow{2}{*}{ Q1 } & \multirow{2}{*}{ Mediana } & \multirow{2}{*}{ Q3 } & \multirow{2}{*}{$\mathrm{Q} 3-\mathrm{Q} 1$} \\
\hline & & & & & Mínimo & Máximo & & & & \\
\hline 7 & 19 & 10,95 & 3,97 & 0,91 & 9,04 & 12,86 & 8 & 11 & 13 & 5 \\
\hline 8 & 12 & 12,17 & 4,55 & 1,31 & 9,28 & 15,06 & 8 & 11,5 & 16,5 & 8,5 \\
\hline 9 & 15 & 15,20 & 4,30 & 1,11 & 12,82 & 17,58 & 12 & 16 & 20 & 8 \\
\hline 10 & 18 & 14,61 & 5,53 & 1,30 & 11,86 & 17,36 & 8 & 15,5 & 20 & 12 \\
\hline 11 & 10 & 15,80 & 4,02 & 1,27 & 12,92 & 18,62 & 14,75 & 16 & 18,5 & 3,75 \\
\hline 12 & 15 & 15,93 & 4,89 & 1,26 & 13,22 & 18,64 & 12 & 18 & 20 & 8 \\
\hline 13 & 13 & 17,77 & 3 & 0,83 & 15,95 & 19,58 & 15 & 20 & 20 & 5 \\
\hline Total & 102 & 14,44 & 4,85 & 0,48 & 13,49 & 15,39 & 10,75 & 15 & 20 & 9,25 \\
\hline
\end{tabular}

A média dos escores considera válidas todas as pontuações obtidas pelos sujeitos. Isto não é compatível com a proposta do teste, já que uma pontuação parcial no subteste Piaget 1 (quando a criança acerta sua mão direita e erra sua mão esquerda, por exemplo) indica a ausência da orientação direita-esquerda em si mesmo, e não uma aquisição parcial. Isto é válido também para os subtestes Piaget 2 (discriminação direita-esquerda no examinador) e Piaget 3 (discriminação direitaesquerda entre os objetos). Por isso, com o objetivo de traçar a curva de desenvolvimento da orientação direita-esquerda na amostra de normatiza- ção, propõe-se utilizar, para os subtestes Piaget 1, 2 e 3, não a média dos escores, mas uma distribuição dos indivíduos em duas categorias quanto à orientação direita-esquerda: AQUISIÇÃO e NÃO AQUISIÇÃO da habilidade. Isto significa considerar, para cada um dos subtestes, somente os casos com pontuação máxima (2 pontos para Piaget 1; 4 pontos para Piaget 2; 14 pontos para Piaget 3).

Por este método, pôde-se chegar a uma curva de desenvolvimento para a orientação direita-esquerda, decorrente da análise dos subtestes Piaget 1, 2 e 3. A Figura 1 resume os dados, comparando-os com os escores equivalentes da normatização francesa. 
Figura 1. Indivíduos com pontuação máx ima nos subtestes Piaget 1, Piaget 2 e Piaget 3 e comparação de medianas do escore Piaget 1-2-3 nas padronizações de 1950 e 2003 da Bateria Piaget-Head.

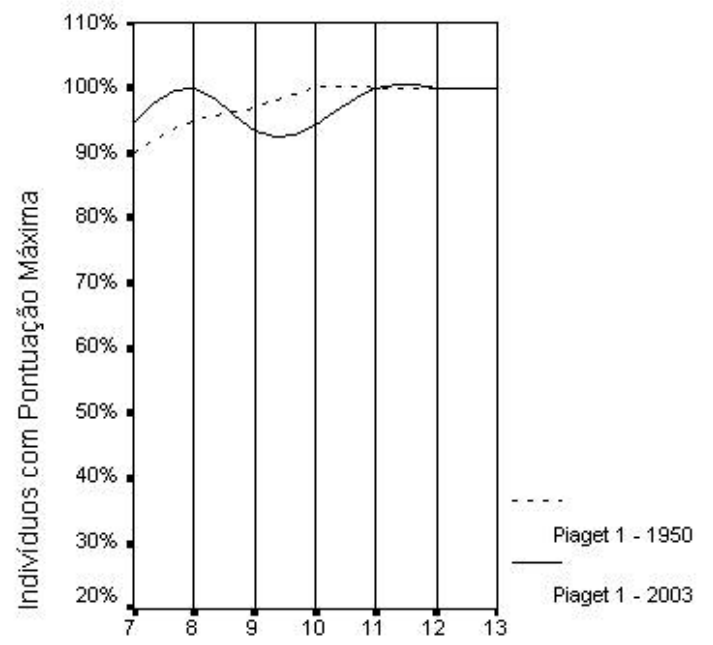

Idade

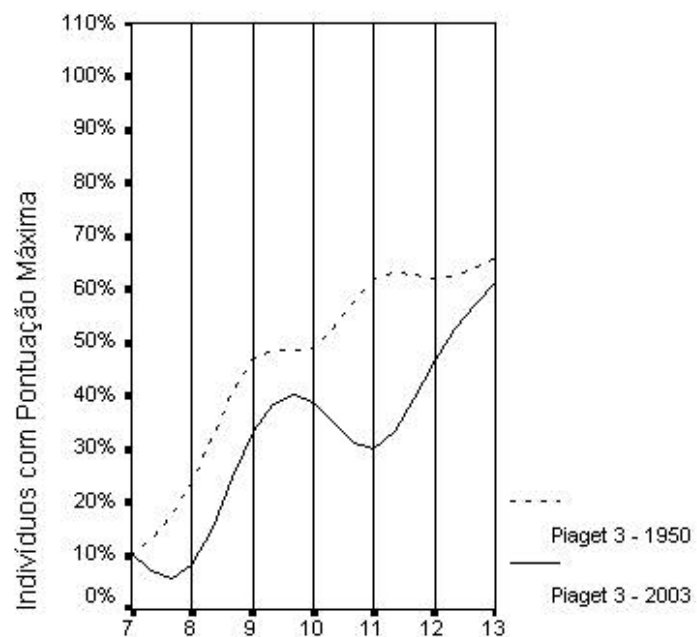

Idade

Pela Figura 1 constata-se que a orientação direita-esquerda em si mesmo (Piaget 1) está adquirida em $95 \%$ das crianças com 7 anos de idade, sendo que este padrão praticamente se mantém nas demais faixas etárias.

Já a orientação direita-esquerda no examinador (Piaget 2) está adquirida por aproximadamente $55 \%$ das crianças com a idade de 7 anos. Apesar da curva mostrar-se ascendente em relação aos grupos etários, existe um pico de desempenho aos 8 anos (100\% das crianças com esta idade) seguido por uma queda da curva de desenvolvimento na faixa etária seguinte (aquisição da

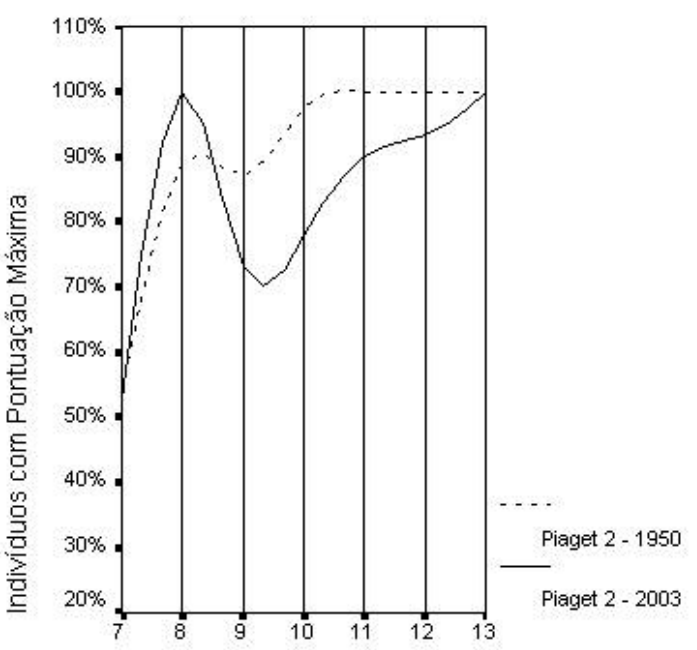

Idade

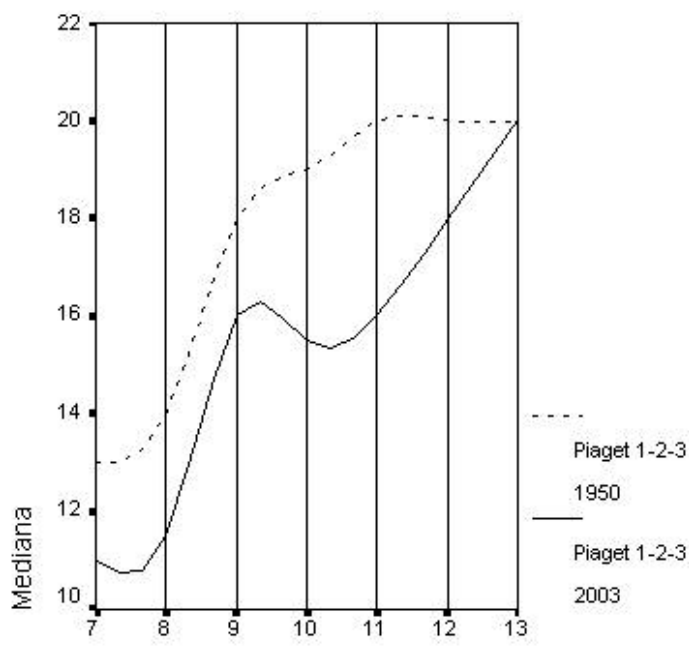

Idade

habilidade em $70 \%$ das crianças com 9 anos). Gradativamente a curva torna a ascender, alcançando os 100\% de indivíduos com a habilidade adquirida somente no grupo de 13 anos.

De forma semelhante, a curva que representa a orientação direita-esquerda entre os objetos (Piaget 3) é ascendente, demonstrando uma aquisição gradativa no decorrer dos anos. Deste modo, $10 \%$ da amostra com a idade de 7 anos e $60 \%$ das crianças com 13 anos alcançaram pontuação máxima na prova. Como no subteste Piaget 2 , houve um retrocesso em $10 \%$ na curva de desenvolvimento (porém, neste caso, entre as idades 
de 10 e 11 anos). Acredita-se que a presença de pontos de flutuação nas curvas apresentadas na Figura 1 esteja relacionada com o tamanho reduzido da amostra para cada faixa etária $(X=14 ; S=3)$. Esta hipótese será testada na continuidade do estudo, com o aumento substancial da amostra de normatização.

Os dados sugerem uma extensão da amostra de normatização a outras faixas etárias já que, por um lado, praticamente todas as crianças de 7 anos obtiveram êxito no Piaget 1 (incluir faixas etárias anteriores) e somente $60 \%$ das crianças com 13 anos tiveram desempenho satisfatório no Piaget 3 (incluir faixas etárias posteriores).

Em relação à normatização francesa da década de 50 (Zazzo, 1981), percebe-se um menor desempenho dos indivíduos que compõe a amostra de normatização do presente estudo, como ilustra a Figura 1. Apesar da impossibilidade de se demonstrar estatisticamente esta diferença (devido à falta dos dados brutos da normatização francesa), a porcentagem de indivíduos que discriminam direita-esquerda no examinador e entre os objetos do ambiente é menor no estudo piloto em comparação com a normatização original. No entanto, desconsiderando as idades de 8 anos (para Piaget 2) e 11 anos (para Piaget 3), nota-se uma equivalência proporcional das curvas de desenvolvimento dos dois estudos normativos. Este fator sugere que as etapas de desenvolvimento da orientação direitaesquerda se mantiveram constantes.

A fidedignidade do teste não se aplica do mesmo modo que em provas de rendimento, já que o objetivo dos itens é verificar se um determinado grau de orientação direita-esquerda foi atingido ou não. Mesmo assim, no subteste que é possível uma análise da precisão (Piaget 3, composto por 6 itens), foi calculado o coeficiente de correlação produto-momento de Pearson entre os resultados obtidos nos itens pares e ímpares. A correlação encontrada $(r=0,65 ; p<0,001)$ é significativa, sugerindo estabilidade no constructo avaliado pelo subteste.

Uma descrição dos percentis para 0 escore Piaget 1-2-3, em função da idade, pode ser visualizada na Tabela 2 .

\section{Tabela 2. Percentis para o Escore Piaget 1-2-3.}

\begin{tabular}{c|c|ccc|c|c|cc}
\hline \multirow{2}{*}{ Idade } & \multirow{2}{*}{$\mathrm{N}$} & \multicolumn{7}{c}{ PERCENTIL } \\
\cline { 3 - 9 } & & 5 & 10 & 25 & 50 & 75 & 90 & 95 \\
7 & 19 & 4 & 6 & 8 & 11 & 13 & 18 & - \\
8 & 12 & 6 & 6,6 & 8 & 11,5 & 16,5 & 19,4 & - \\
9 & 15 & 6 & 8,4 & 12 & 16 & 20 & 20 & - \\
10 & 18 & 4 & 5,8 & 8 & 15,5 & 20 & 20 & - \\
11 & 10 & 6 & 6,8 & 14,8 & 16 & 18,5 & 20 & - \\
\hline \hline 12 & 15 & 6 & 7,2 & 12 & 18 & 20 & 20 & - \\
\hline 13 & 13 & 13 & 13 & 15 & 20 & 20 & 20 & - \\
\hline
\end{tabular}




\section{Considerações finais}

Como salientam Noronha, Freitas e Ottati (2002), a revisão periódica de um teste é fundamental para que ele continue oferecendo a confiabilidade necessária a qualquer instrumento psicológico. Na realidade, o uso indevido de testes não padronizados à população local põe em risco a própria validade de uma avaliação psicológica que se utilize deles. Nesse sentido, a presente pesquisa teve como finalidade estimar normas locais para a Bateria Piaget-Head, um teste que, apesar de bastante utilizado em avaliações neuropsicológicas, por muitos anos foi negligenciado em seus aspectos psicométricos. Na continuidade do estudo, um aumento substancial da amostra de normatização possibilitará também a estimação de parâmetros psicométricos para a Bateria PiagetHead, como unidimensionalidade, dificuldade dos itens, precisão e validade. Por ora, estes resultados forneceram indícios que sugerem: uma mesma curva de desenvolvimento para a orientação direita-esquerda, desconsiderando diferenças de gênero, escola e lateralidade; o acréscimo das idades de 6 e 14 anos na amostra de normatização. Mesmo que preliminar, o presente estudo caracterizou-se por ser a primeira normatização brasileira da Bateria Piaget-Head, contribuindo para o aumento da confiabilidade das avaliações que se utilizam do referencial psicométrico.

\section{Referências}

Conselho Federal de Psicologia. (2003). Resolução 002/ 2003 - Critérios de avaliação da qualidade de testes psicológicos. Brasília: Conselho Federal de Psicologia.

Fonseca, V. (1995). Introdução às dificuldades de aprendizagem. Porto Alegre: Artes Médicas.

Hutt, E., \& Hutt, S. J. (1974). Observação direta e medida do comportamento. São Paulo: EPU.

Kroeff, P. (1998). Normas para o Teste de Bender. Psicologia: Reflexão e Crítica, 3(1/2), 12-19.
Lefèvre, B. (1998). Avaliação neuropsicológica do adulto. In: Capovilla, F. C., Gonçalves, M. J., \& Macedo, E. C. (orgs.). Tecnologia em (re)habilitação cognitiva: Uma perspectiva multidisciplinar. São Paulo: EDUNISC.

\section{Luria, A. R. (1981). Fundamentos de neuropsi-} cologia. São Paulo: EDUSP.

Noronha, A. P. P., \& Alchieri, J. C. (2002). Reflexões sobre os instrumentos de avaliação psicológica. In: Primi, R. (org.) Temas em avaliação psicológica. Campinas: Instituto Brasileiro de Avaliação Psicológica.

Noronha, A. P. P., Freitas, F. A., \& Ottati, F. Parâmetros psicométricos de testes psicológicos de inteligência. Interação em Psicologia, 6(2), 195201.

Pamplona Morais, A. M. (1992). Distúrbios da Aprendizagem: Uma abordagem psicopedagógica. São Paulo: EDICON.

Riechi, T. I. J. S. Uma proposta de leitura neuropsicológica dos problemas de aprendizagem. Curitiba, 1996. Dissertação de Mestrado, Universidade Federal do Paraná.

Riechi, T. I. J. S., Kruszielski, L, Marins, M. C., \& De Toni, P. M. (2002). Práticas psicopedagógicas: Uma abordagem neuropsicológica. Curitiba: edição dos autores.

Simões, M. R. (2002). Avaliação neuropsicológica em crianças e adolescentes. In: Primi, R. (org.) Temas em avaliação psicológica. Campinas: Instituto Brasileiro de Avaliação Psicológica.

Spss, Inc.(1994). SPSSwindows user's guide. New York: MacGraw Hill.

Zazzo, R. (1981). Manual para o exame psicológico da criança. São Paulo: Mestre Jou.

Recebido em/ received in: 24/03/2004 Aprovado em/ approved in: 08/04/2005 